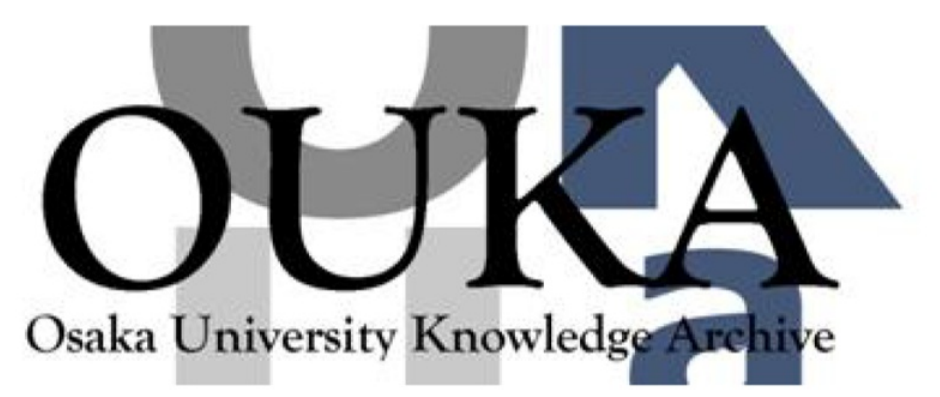

\begin{tabular}{|c|l|}
\hline Title & $\begin{array}{l}\text { Optical properties of self-assembled thin-fi lm } \\
\text { of poly(p-phenylene vinylene)s and its } \\
\text { application to light-emitting devices with } \\
\text { microring geometry }\end{array}$ \\
\hline Author(s) & Sonoda, T.; Fujisawa, T.; Fuji i, A. et al. \\
\hline Citation & Applied Physics Letters. 76(22) p. 3227-p. 3229 \\
\hline Issue Date & $2000-05-25$ \\
\hline oaire:version & VoR \\
\hline URL & https://hdl. handle.net/11094/75653 \\
\hline rights & \\
\hline Note & \\
\hline
\end{tabular}

Osaka University Knowledge Archive : OUKA

https://ir. Library. osaka-u. ac. jp/

Osaka University 


\section{Optical properties of self-assembled thin- film of poly(p-phenylene vinylene)s and its application to light-emitting devices with microring geometry}

Cite as: Appl. Phys. Lett. 76, 3227 (2000); https://doi.org/10.1063/1.126589

Submitted: 14 February 2000 . Accepted: 06 April 2000 . Published Online: 25 May 2000

T. Sonoda, T. Fujisawa, A. Fujii, and K. Yoshino

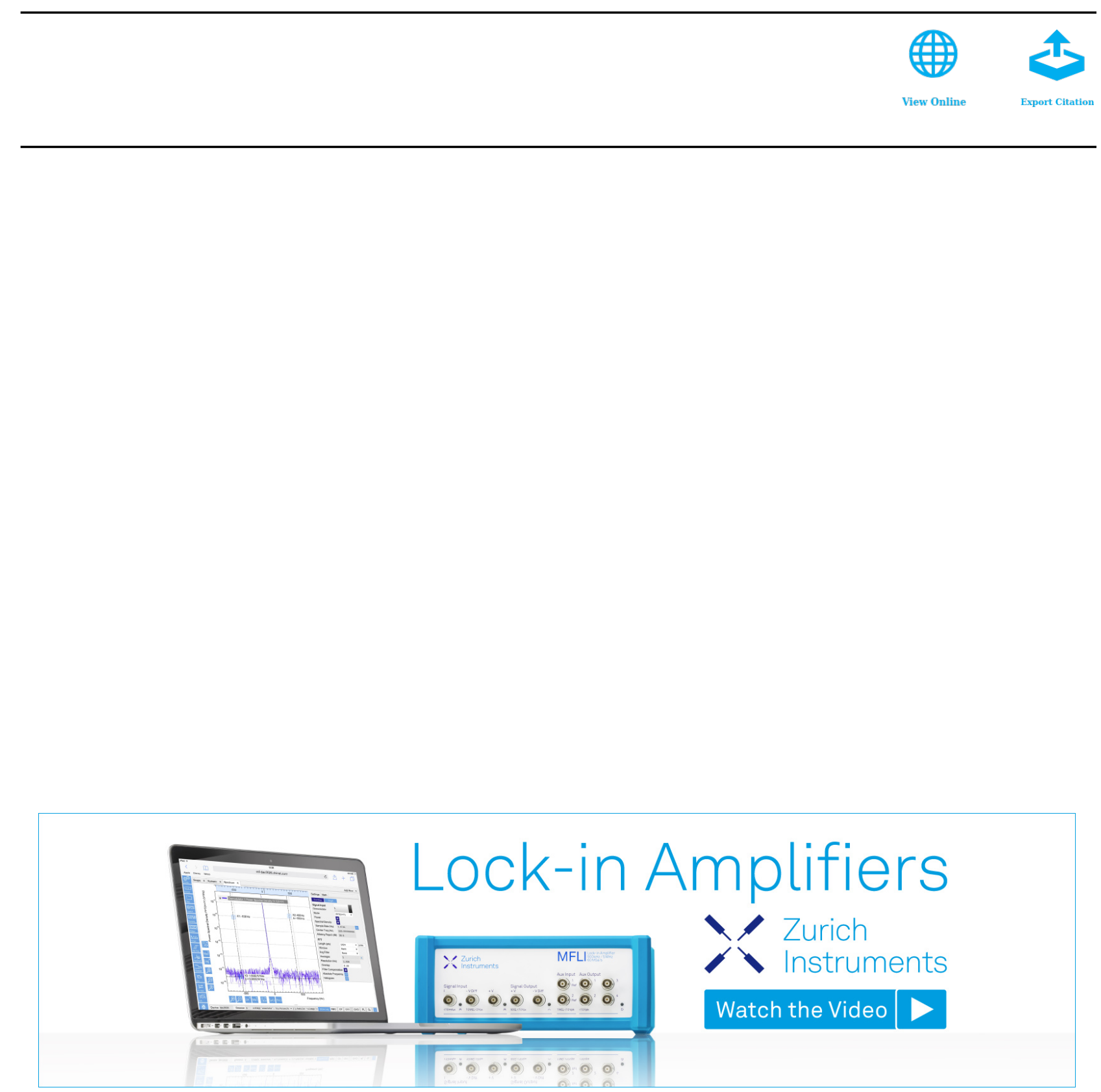




\title{
Optical properties of self-assembled thin-film of poly $(p$-phenylene vinylene)s and its application to light-emitting devices with microring geometry
}

\author{
T. Sonoda, T. Fujisawa, A. Fujii, and K. Yoshino ${ }^{a)}$ \\ Department of Electronic Engineering, Graduate School of Engineering, Osaka University, \\ 2-1 Yamada-oka, Suita, Osaka 565-0871, Japan
}

(Received 14 February 2000; accepted for publication 6 April 2000)

\begin{abstract}
Periodic multilayer structures of poly( $p$-phenylene vinylene)s have been fabricated by a self-assembly method on flat surfaces and round surfaces of optical fibers. Alternating multilayers consisting of poly\{1,4-[2-(5-carboxypentyloxy)-5-methoxyphenylene]vinylene $\}$ (CPMOPPV) and poly ( $p$-phenylene vinylene) (PPV) were adsorbed onto the positively charged substrates. The optical properties of the periodic multilayer structures of CPMOPPV/PPV have been studied. Periodic multilayers with microring geometry have also been fabricated around the quartz fibers. Their optical properties have been studied, and yellow electroluminescence from a light-emitting device with microring geometry has been observed. (C) 2000 American Institute of Physics.
\end{abstract}

[S0003-6951(00)03822-5]

Conducting polymers with highly extended conjugated $\pi$-electron systems in the main chains have attracted great interest from both fundamental and practical viewpoints, because they exhibit various novel properties such as insulator-metal transition upon doping, ${ }^{1}$ electroluminescence (EL), ${ }^{2,3}$ photovoltaic effect. ${ }^{4}$

A self-assembly method is one of the important techniques to fabricate ultrathin polymer film or polymer multilayer film controlled in molecular level., ${ }^{5,6}$ In the selfassembly method, charged substrates are repeatedly dipped in cation and anion solutions alternately and alternating multilayers of the cation and anion are formed by Coulombic force between cation and anion on the substrates. Utilizing various combination of many kinds of cation and anion materials, various self-assembled multilayers utilizing conducting polymers could be fabricated and their applications were demonstrated. Previously, we found the efficient photoinduced charge separation at interfaces of self-assembled conducting polymers, and suggested the efficient photocells as one of the applications. ${ }^{7,8}$ EL device with self-assembled organic multilayers is also one of the most attractive applications and has a possibility to realize a flat panel display. ${ }^{9}$

Poly( $p$-phenylene vinylene) (PPV) and its derivatives are the promising materials, because of their high luminescent quantum efficiency. Recently, we have developed a PPV derivative, poly\{1,4-[2-(5-carboxypentyloxy)-5methoxyphenylene]vinylene $\}$ (CPMOPPV), for the selfassembly method, and studied its optical and electrical properties. ${ }^{10}$ Since this polymer indicates the characteristics of a polyanion, self-assembled alternating multilayer structures based on PPVs could be formed utilizing PPV precursor as a polycation. ${ }^{11}$

On the other hand, polymer lasers with microring structure have attracted much attention as a novel type of lasers. By dipping an optical fiber into a conducting polymer solution, a cylindrical microring structure consisting of the con-

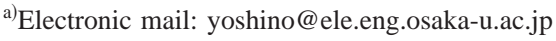

ducting polymer was easily formed around the fiber. Recently, we have observed the laser emission from the microring structure of poly(2,5-dialkoxy- $p$-phenylene vinylene) (ROPPV). ${ }^{12}$ Furthermore, EL devices with microcavity structure have been fabricated utilizing several kinds of conducting polymers. ${ }^{13,14}$ Since the self-assembled multilayers can be formed onto substrates with any shapes due to Coulombic force, it should be expected to fabricate selfassembled cylindrical multilayers onto round and small-sized substrates such as optical fibers.

In this letter, periodic multilayer structures consisting of PPV and CPMOPPV have been fabricated onto flat substrates and optical fibers by self-assembly method. The optical properties of the periodic multilayer structures of PPVs and light-emitting devices (LED) with the microring geometry around the optical fibers have been studied.

The molecular structures of PPV precursor, PPV, and CPMOPPV are shown in Fig. 1. Preparation of and PPV precursor and CPMOPPV were reported previously. ${ }^{10,11}$ CPMOPPV is soluble in common organic solvents such as acetone, ethanol, and tetrahydrofuran. The sodium salt of CPMOPPV, which is obtained by adding sodium hydroxide, is soluble even in water. PPV could be obtained by annealing PPV precursor at $200^{\circ} \mathrm{C}$ for $6 \mathrm{~h}$ in vacuum. CPMOPPV and PPV precursor were used as a polyanion and a polycation, respectively.

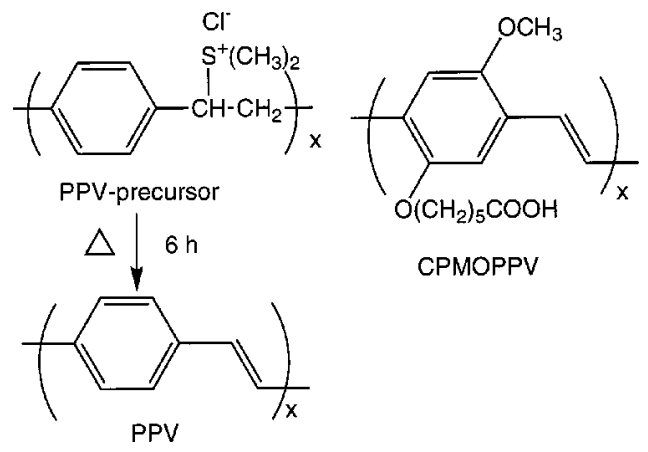

FIG. 1. The molecular structures of PPV-precursor, PPV, and CPMOPPV. 


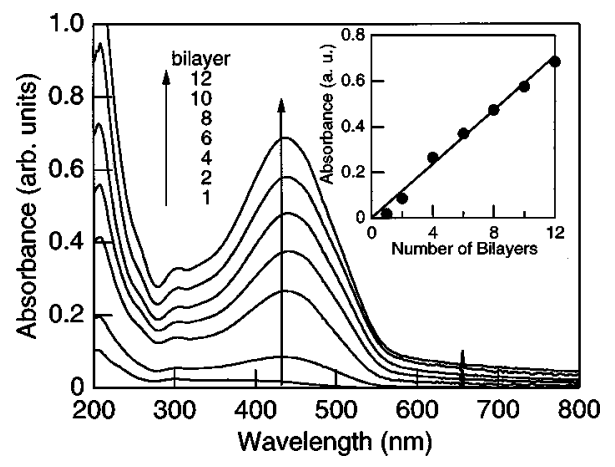

FIG. 2. Absorption spectra of the CPMOPPV/PPV multilayer structures. The inset shows the dependence of the absorbance at $430 \mathrm{~nm}$ on the number of bilayers in the CPMOPPV/PPV multilayer structures.

The fabrication of periodic multilayer structure is described as follows: precleaned flat quartz substrates or quartz fibers were ultrasonicated in acetone for $1 \mathrm{~h}$ and immersed into a toluene solution of $\mathrm{N}$-(2-aminoethyl-3aminopropyl)trimethoxy silane (TMS) of 5 vol \% for $15 \mathrm{~h}$. Then, the substrates or fibers were heated in toluene at $50^{\circ} \mathrm{C}$ for $1 \mathrm{~h}$ and washed with toluene, a toluene-methanol mixture (1:1) and methanol to remove the excess TMS. Besides, the substrates or fibers were ultrasonicated in distilled water for $10 \mathrm{~min}$ and dried at $55^{\circ} \mathrm{C}$ in air for $30 \mathrm{~min}$. The substrates or fibers were thus coated by TMS monolayer charged positively. A methanol solution of CPMOPPV in concentration of $0.01 \mathrm{M}$ were filtered through $0.5 \mu \mathrm{m}$ filter prior to use. The substrates or fibers were immersed into a methanol solution of CPMOPPV for $15 \mathrm{~min}$ in order to form the first adsorbed layer of CPMOPPV. Then, the substrates or fibers were washed extensively with methanol and distilled water in sequence. A PPV precursor layer was formed by immersing the substrates or fibers into an aqueous solution of PPV precursor $(0.01 \mathrm{M})$ for $15 \mathrm{~min}$, and then washed with distilled water and methanol in sequence. By repeating the processes, the multilayer structures of CPMOPPV/PPV precursor were fabricated on the substrates or fibers. CPMOPPV/ PPV multilayer structures were obtained by thermal conversion of the CPMOPPV/PPV-precursor multilayer structures.

Absorption spectra and photoluminescence (PL) spectra of the CPMOPPV/PPV multilayer structures were measured using a Hewlett Packard HP8452A spectrophotometer and a Hitachi F-4500 fluorescence spectrophotometer, respectively. X-ray diffraction ( $\mathrm{CuK} \alpha$ line) measurement was done by a Rigaku RINT 1100 diffractometer. CPMOPPV/ PPV multilayers were also fabricated around $125 \mu \mathrm{m}$ diameter of indium-tin-oxide (ITO) coated glass fibers to make LEDs with microring geometry. After the fabrication of CPMOPPV/PPV multilayers, aluminum (Al) were evaporated onto half of the fibers to form semicylindrical electrodes by vacuum deposition under the vacuum of about $10^{-6}$ Torr. EL spectra were measured using conventional methods and all of the EL measurements were carried out in vacuum at room temperature.

Figure 2 shows the optical absorption spectra of CPMOPPV/PPV multilayers depending on the number of bilayers. The absorption peak around $430 \mathrm{~nm}$ is corresponding to the $\pi-\pi^{*}$ transition of CPMOPPV and PPV, therefore

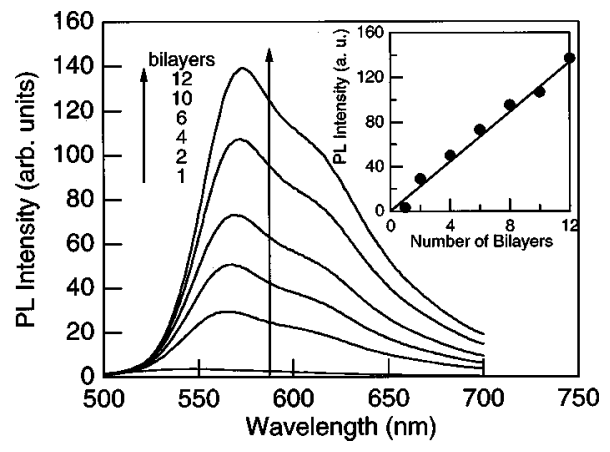

FIG. 3. PL spectra of the CPMOPPV/PPV multilayer structures. The inset shows the dependence of the PL intensity at $570 \mathrm{~nm}$ on the number of bilayers in the CPMOPPV/PPV multilayer structures.

the conversion from CPMOPPV/PPV-precursor multilayers to CPMOPPV/PPV multilayers was successfully performed, the detailed studies of which were reported elsewhere. ${ }^{11}$ The absorption spectra of CPMOPPV/PPV multilayers were essentially superposition of those of CPMOPPV and PPV. The inset of Fig. 2 shows the absorbance of CPMOPPV/PPV multilayers at $430 \mathrm{~nm}$ depending on the number of bilayers, and the absorption of CPMOPPV/PPV multilayers increased in proportion to the number of bilayers, which indicates that the thickness of each bilayer is almost constant.

The PL spectra of CPMOPPV/PPV multilayers are shown in Fig. 3, when the $430 \mathrm{~nm}$ blue light from Xe lamp is adopted as the excitation source. The PL peak is corresponding to the radiative recombination of excitons or exciton polarons in the main chains of CPMOPPV,,$^{10}$ and the slight red-shift of PL peak depending on the number of bilayers was observed. Such red-shifts of PL peak in self-assembled thin films were previously reported. ${ }^{15}$ The PL intensity of CPMOPPV/PPV multilayers markedly increased with increasing the number of bilayers. As is apparent in the inset of Fig. 3, the linear relationship was observed between the PL intensity at $570 \mathrm{~nm}$ and the number of bilayers. Such results also exhibit that constant amounts of the polymers were adsorbed by the immersion processes, that is, the PL intensity as well as the absorbance shows the relative thickness of the polymers.

$\mathrm{X}$-ray diffraction pattern of the eight-bilayer film of the CPMOPPV/PPV multilayer was shown in Fig. 4. The several broad peaks were observed in the figure. Such peaks were

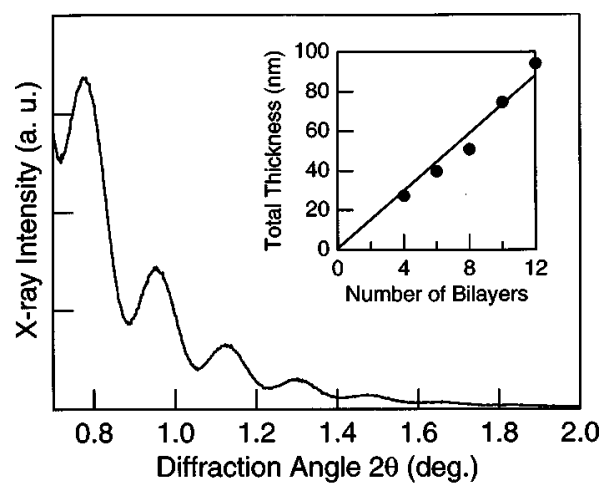

FIG. 4. X-ray diffraction pattern of an eight-bilayer film of CPMOPPV/PPV multilayer structure. The inset shows the dependence of the film thickness on the number of bilayers. 


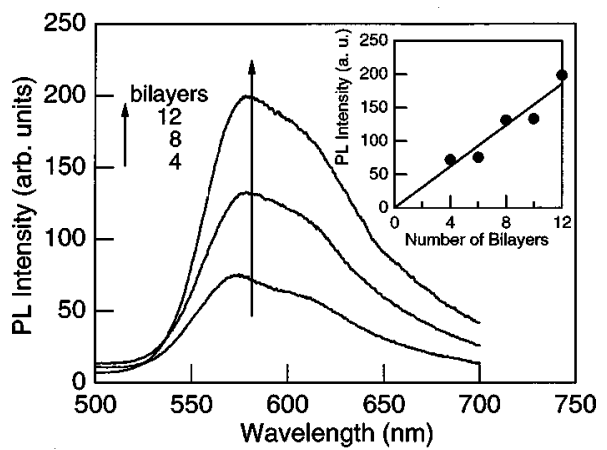

FIG. 5. PL spectra of the CPMOPPV/PPV multilayer structures with microring geometry. The inset shows the dependence of the PL intensity at 580 $\mathrm{nm}$ on the number of bilayers in the CPMOPPV/PPV multilayer structures with microring geometry.

also observed in CPMOPPV/PPV multilayers with different number of bilayers. The peaks must be attributed to the total thickness of the thin films, which might imply that the film surface is quite smooth. The film thickness could be evaluated from the differential between the peaks and increased linearly depending on the number of bilayers, as shown in the inset of Fig. 4. Therefore, the thickness of each bilayer is roughly estimated to be $7 \mathrm{~nm}$.

Ultrathin and smooth films can be fabricated on the substrates with any shapes by utilizing the self-assembly method, so we tried to fabricate CPMOPPV/PPV multilayers around $125-\mu \mathrm{m}$-diam quartz fibers. Figure 5 shows the PL spectra of CPMOPPV/PPV multilayers around the quartz fibers, when the wavelength of the excitation light was 430 $\mathrm{nm}$. The spectra of CPMOPPV/PPV multilayers around the quartz fibers were similar to those of the flat thin film on the quartz substrate as shown in Fig. 3. The inset of Fig. 5 shows the PL intensity at $580 \mathrm{~nm}$ increased linearly as a function of the number of bilayers. The linear increment of the PL intensity depending on the number of bilayers indicates that alternating layers of CPMOPPV and PPV were formed even around the quartz fibers.

The LEDs with CPMOPPV/PPV multilayer structure with cylindrical geometry are the novel trial for the realization of the polymer lasers, which means that cylindrical geometry of the polymers acts as a microcavity for ring lasers. The LEDs, which consist of ITO/(CPMOPPV/PPV) $)_{\mathrm{n}} / \mathrm{Al}$, could be fabricated successfully, the device structure of which is shown in the inset of Fig. 6.

Strong yellow EL was observed from the LED with microring geometry. The typical EL spectrum at $14 \mathrm{~V}$ of the applied voltage is shown in Fig. 6, and coincides with that of LED with the flat thin film on ITO coated glass substrate. ${ }^{11}$ The emission intensity starts to increase at around $10 \mathrm{~V}$, and increases monotonically with increasing injection current. It is important to have shown that the LEDs with the microring geometry can be easily fabricated, and electrical or optical devices with self-assembled alternating multilayers on the unique shape substrates might be realized in the near future. The microring lasers with self-assembled multilayers should be one of the most impressive topics. The detailed studies to clarify the laser characteristics of CPMOPPV/PPV multilayers with microring geometry are now in progress.

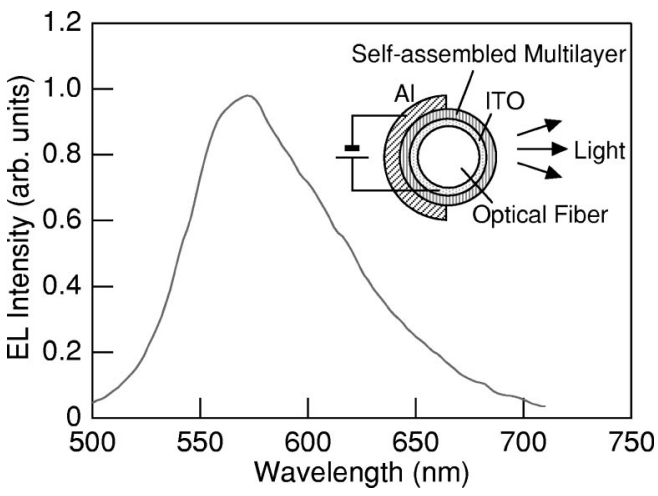

FIG. 6. EL spectrum of the microring LED with six bilayers of the CPMOPPV/PPV multilayer at room temperature in vacuum. The inset shows the device structure of LED.

In summary, the periodic multilayer structures based on poly $(p$-phenylene vinylene)s were fabricated successfully by the self-assembly method. The alternating multilayers of CPMOPPV/PPV were formed onto the positively charged substrates, and the adsorbed multilayers were confirmed by the absorption and PL measurements. Periodic multilayers of CPMOPPV/PPV were also fabricated successfully around the quartz fibers and PL depending on the number of bilayers was observed as well as the flat thin films on the quartz substrates. The LEDs with the microring geometry were fabricated around fibers and yellow electroluminescence was observed.

This work was partly supported by the Research for the Future Program of the Japan Society for the Promotion of Science (Project No. JSPS-RFTF96P00206) and by a Grantin-Aid for Scientific Research from Japan Society for the Promotion of Science.

${ }^{1}$ A. J. Heeger, S. Kivelson, J. R. Schrieffer, and W. P. Su, Rev. Mod. Phys. 60, 781 (1988).

${ }^{2}$ J. H. Burroughes, D. D. C. Bradley, A. R. Brown, R. N. Marks, K. Mackay, R. H. Friend, P. L. Burns, and A. B. Holmes, Nature (London) 347, 539 (1990).

${ }^{3}$ Y. Ohmori, M. Uchida, K. Muro, and K. Yoshino, Jpn. J. Appl. Phys., Part 2 30, L1941 (1991).

${ }^{4}$ S. Morita, A. A. Zakhidov, and K. Yoshino, Solid State Commun. 82, 249 (1992).

${ }^{5}$ G. Decher, J. D. Hong, and J. Schmitt, Thin Solid Films 210/211, 831 (1992).

${ }^{6}$ M. Ferreira, J. H. Cheung, and M. F. Rubner, Thin Solid Films 244, 806 (1994).

${ }^{7}$ T. Kawai, T. Yamaue, K. Tada, M. Onoda, S-H. Jin, S-K. Choi, and K. Yoshino, Jpn. J. Appl. Phys., Part 2 35, L741 (1996).

${ }^{8}$ T. Yamaue, T. Kawai, M. Onoda, and K. Yoshino, Jpn. J. Appl. Phys., Part 1 38, 6008 (1999).

${ }^{9}$ M. Onoda and K. Yoshino, Jpn. J. Appl. Phys., Part 2 34, L260 (1995).

${ }^{10}$ A. Fujii, T. Sonoda, and K. Yoshino, Jpn. J. Appl. Phys., Part 2 39, L249 (2000).

${ }^{11}$ T. Sonoda, A. Fujii, and K. Yoshino, Jpn. J. Appl. Phys., Part 1 (to be published).

${ }^{12}$ S. V. Frolov, M. Shkunov, Z. V. Vardeny, and K. Yoshino, Phys. Rev. B 56, R4363 (1997).

${ }^{13}$ A. Fujii, S. V. Frolov, Z. V. Vardeny, and K. Yoshino, Jpn. J. Appl. Phys., Part 2 37, L740 (1998).

${ }^{14}$ S. V. Frolov, A. Fujii, D. Chinn, M. Hirohata, R. Hidayat, M. Teraguchi, T. Masuda, K. Yoshino, and Z. V. Vardeny, Adv. Mater. 10, 869 (1998).

${ }^{15}$ H. Hong, M. Tarabia, H. Chayet, D. Davidov, E. Z. Faraggi, Y. Avny, R. Neumann, and S. Kirstein, J. Appl. Phys. 79, 3082 (1996). 